\title{
Analysis of the ionospheric parameter dynamics on the basis of a generalized multicomponent model
}

\author{
Oksana Mandrikova ${ }^{1}$, Nadezhda Fetisova ${ }^{1}$, Yuryi Polozov ${ }^{1}$, and Vladimir Geppener ${ }^{2}$ \\ ${ }^{1}$ Institute of Cosmophysical Research and Radio Wave Propagation FEB RAS, 684034, \\ Kamchatskykrai, Paratunka, Russian Federation \\ ${ }^{2}$ Sankt-Petersburg Electrotechnical University “LETI”, 197376, Saint-Petersburg, Russian Federation
}

\begin{abstract}
In the present paper, we have carried out an analysis of the ionospheric critical frequency data of the F2 layer during strong magnetic storms in 2017-2018. The ionospheric data of Paratunka (IKIR FEB RAS, Kamchatka, 53.0 N, 158.7 E), Wakkanai (Japan, 45.16 N, 141.75 E), and Moscow stations (Russia, 55.49 N, 37.29 E) were used. The study was carried out using a generalized multicomponent model (GMCM) developed by the authors. GMCM allows studying the dynamics of the ionospheric parameters in detail and estimating their characteristics. Using the modeling, we detected and studied anomalous changes in the ionosphere preceding and accompanying the periods of magnetic storms in the analyzed areas. The study results were compared with the traditional median method and showed the perspectiveness of GMCM. The research is supported by a grant from the Russian Science Foundation (project No. 14-11-00194).
\end{abstract}

\section{Introduction}

Ionospheric response to the near-Earth space state has a complex nature, many aspects of which have not been sufficiently studied $[1,2,3]$. The recorded ionospheric parameters include regular diurnal and seasonal changes, and their dynamics depends on solar and geomagnetic activity, geographic location (polar and auroral zones, mid-latitude and equatorial regions), and on other factors $[1,2,3]$. The most severe irregular changes are observed in the ionosphere during solar flare events and magnetic disturbances and lead to the formation of ionospheric irregularities (ionospheric storms). They often occur in the equatorial and auroral zones $[1,2,3]$, but can also be observed in the mid-latitudes during disturbed periods $[4,5,6]$. Ionospheric storms can include positive and negative phases, having a complex space-time distribution [2, 3, 4]. Positive (negative) phases are characterized by an anomalous increase (decrease) in the electron concentration of the ionosphere, which has negative impact on modern technical system operation. A number of studies [3, 7-9] have noted that positive phases of ionospheric storms can be observed on the eve of strong magnetic storms. Timely detection of anomalous effects has important scientific and applied significance $[1,3,10]$. 
The main approaches and methods for studying of ionospheric processes include the traditional moving median method [1, 11], ionosphere physical models [12], ionosphere empirical models [13, 14], and neural network methods and models [2, 15]. A complex non-stationary nature of the ionospheric parameters impedes the application of traditional methods [16], which allow describing long-term changes of the ionospheric parameters but lead to a loss of information on ionospheric irregularities dynamics [4]. The accuracy and efficiency of modern methods (empirical, physical and neural networks) depend on the presence of qualitative operative data (the indices of magnetic and solar activities, the solar ultraviolet radiation spectrums, the atmospheric parameters, etc. are used) and are significantly reduced during disturbed periods due to the gaps in data, increase of the interference level, and so on. Therefore, intensive development of technologies for monitoring of the near-Earth space state and methods of data analysis is currently observed $[17,18]$.

In this work, analysis of the ionospheric parameter dynamics was carried out during magnetic storms in 2017 - 2018 (the ionospheric critical frequency data (foF2) of Paratunka (Russia, 53.0 N, 158.7 E), Wakkanai (Japan, 45.16 N, 141.75 E), and Moscow (Russia, $55.49 \mathrm{~N}, 37.29$ E) stations were analyzed, and we used the resources http://spidr.ionosonde.net/spidr and http://wdc.nict.go.jp). The analysis was performed using a generalized multicomponent model (GMCM) developed by the authors. GMCM identification is based on a complex approach, combining the wavelet transform methods and ARIMA models [4]. The proposed model allows describing regular time course of the ionospheric parameters and estimating anomalous changes occurring during ionospheric storms. In this paper, the estimation of GMCM parameters was carried out for 15-minute data of the ionospheric critical frequency (we used the data of Paratunka station). Based on the obtained model, we detected and studied anomalous changes in the ionospheric parameters, occurring during magnetic storms.

\section{Generalized multicomponent model of ionospheric parameters}

The ionospheric parameter time series includes regular and anomalous components and can be represented as generalized multicomponent model (GMCM) [19]:

$$
f(t)=A^{R E G}(t)+I(t)+e(t)=\sum_{\mu=\overline{1, T}} \alpha^{\mu}(t)+\sum_{\eta} \delta_{\text {dist }}^{\eta}(t)+e(t)
$$

where $A^{R E G}(t)=\sum_{\mu=\overline{1, T}} \alpha^{\mu}(t)(\mu=\overline{1, T}$ is the component number) is a model component describing ionospheric parameter regular variations (during the periods of absence of disturbances in the ionosphere), and including $\alpha^{\mu}(t)$ components, determined by the level of solar activity, geomagnetic conditions and data recording location (geographical coordinates); $I(t)=\sum_{\eta} \delta_{\text {dist }}^{\eta}(t)$ is an anomalous component including $\delta_{\text {dist }}^{\eta}(t)$ multiscale components, having random nature and occurring during ionospheric disturbances (in case of regular data changes, it is assumed that $I(t)=0) ; e(t)$ is a random component including artificial noise (rocket launch, industrial bursts and so on) as well as hardware failures etc.

2.1 Using the multiresolution wavelet decompositions (MRA [20]) and ARIMA methods [16], the $A^{R E G}$ regular component can be represented in the parametric form [4, 21]:

$$
A^{R E G}(t)=\sum_{\mu=\overline{1, T}} \sum_{k=1, N_{j}^{\mu} \text { reg }} s_{j}^{\mu} \text { reg }, k_{j} b_{j r e g}^{\mu}(t)
$$


where $s_{j^{\text {reg }}, k}^{\mu}=\sum_{l=1}^{p_{j^{\text {reg }}}^{\mu}} \gamma_{j^{\text {reg }}, l}^{\mu} \omega_{j^{\text {reg }}, k-l}^{\mu}-\sum_{n=1}^{h_{j^{\text {reg }}}^{\mu}} \theta_{j^{\text {reg }}, n}^{\mu} a_{j^{\text {reg }}, k-n}^{\mu}$ is an estimated value of regular $\mu-$ th component; $p_{j^{\text {reg }}}^{\mu}$ and $\gamma_{j^{\text {reg }}, l}^{\mu}$ are the order and parameters of the $\mu$-th component autoregression; $h_{j^{\text {reg }}}^{\mu}$ and $\theta_{j^{\text {pex }, n}}^{\mu}$ are the order and parameters of a moving average of the $\mu$-th component; $\omega_{j^{\text {reg }}, k}^{\mu}=\nabla^{v^{\mu}} \beta_{j^{\text {reg }}, k}^{\mu}, V^{\mu}$ is the difference order of the $\mu$-th component, $\quad \beta_{j^{r e g}, k}^{l}=c_{j^{r e g}, k}, \quad \beta_{j^{r e g}, k}^{\mu}=d_{j^{r e g}, k}, \mu=\overline{2, T}, \quad c_{j}$ reg $_{, k}=\left\langle f, \phi_{j}\right.$ reg $\left._{, k}\right\rangle, d_{j^{r e g}, k}=$

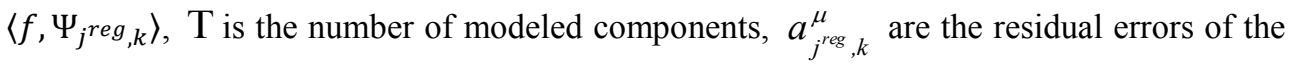
$\mu$-th component model, $N_{j^{\text {reg }}}^{\mu}$ is the length of the $\mu$-th component, $b_{j^{\text {reg }}, k}^{l}=\phi_{j^{\text {reg }}, k}$ is a scaling function, $b_{j^{r e g}, k}^{\mu}=\Psi_{j^{r e g}, k}, \mu=\overline{2, T}$ is the wavelet basis of the $\mu$-th component.

Using the identification method described in the papers [4, 21], we carried out the estimation of the $A^{R E G}$ regular component parameters (see Eq. (2)) for 15-minute data of the critical frequency (foF2). During identification, we used the foF2 data of Paratunka station $(53.0 \mathrm{~N}, 158.7 \mathrm{E})$ from 2016 to 2018 . To approximate the regular time course of the ionospheric parameters, the data recorded during calm geomagnetic conditions (there were no geomagnetic storms), and without strong seismic events in Kamchatka, were used as estimates. Considering the seasonality of ionospheric processes, the foF 2 data of different seasons were modeled separately. The identification results showed that for 15-minute foF 2 data of Kamchatka, the $A^{R E G}$ regular component has the following form:

$$
A^{R E G}(t)=\sum_{\mu=1,2} \sum_{k=1, N_{-5}^{\mu}} s_{-5, k}^{\mu} b_{-5, k}^{\mu}+e(t)
$$

where $s_{-5, k}^{\mu}(t)=\sum_{l=1}^{2} \gamma_{-5, l}^{\mu} \omega_{-5, k-l}^{\mu}+a_{-5, k}^{\mu}(t), \mu=1,2, j=-5$ corresponds to the 5 -th level of the wavelet decomposition (the decomposition level was determined using the algorithm described in the paper [4]).

The model parameters for summer season:

$$
\begin{gathered}
s_{-5, k}^{1}=-0.83 \cdot \omega_{-5, k-1}^{1}-0.84 \cdot \omega_{-5, k-2}^{1}+a_{-5, k}^{1}(t) \\
s_{-5, k}^{2}=-0.86 \cdot \omega_{-5, k-1}^{2}-0.79 \cdot \omega_{-5, k-2}^{2}+a_{-5, k}^{2}(t) .
\end{gathered}
$$

The model parameters for winter season:

$s_{-5, k}^{1}=-0.96 \cdot \omega_{-5, k-1}^{1}-0.97 \cdot \omega_{-5, k-2}^{1}+a_{-5, k}^{1}(t)$;

$s_{-5, k}^{2, k}=-0.96 \cdot \omega_{-5, k-1}^{2}-0.94 \cdot \omega_{-5, k-2}^{2}+a_{-5, k}^{2}(t)$.

2.2 In view of the essential nonstationarity of the function $I(t)=\sum_{\eta} \delta_{\text {dist }}^{\eta}(t)$ (see. Eq. (1)), and following the paper [22], its identification was carried out on the basis of nonlinear approximating schemes. Then we have the following relation:

$I(t)=\sum_{\eta} \delta_{\text {dist }}^{\eta}(t)=\sum_{(\eta, n) \in P_{M}} d_{\eta, n} \Psi_{\eta, n}(t)+\sum_{(\eta, n) \notin P_{M}} d_{\eta, n} \Psi_{\eta, n}(t)=I_{M}(t)+e(t),(3)$ where $I_{M}(t)=\sum_{(\eta, n) \in P_{M}}\left\langle f \Psi_{\eta, n}\right\rangle \Psi_{\eta, n}(t)$ is the projection of $I(t)$ on $M$ vectors, the indices of which are contained in some set $P_{M}$; the component $e(t)=\sum_{\eta, n \notin P_{M}}\left\langle f, \Psi_{\eta, n}\right\rangle \Psi_{\eta, n}$ is a sequence of noise factor affect (assumed that this component is uncorrelated and additive).

In this case, the separation of $I_{M}(t)$ components can be based on the use of threshold functions: 


$$
\sum_{\eta} \delta_{d i s t}^{\eta}(t)=I_{M}(t)=\left\{\begin{array}{c}
\sum_{\eta, n} d_{\eta, n} \Psi_{\eta, n}(t), i f\left|d_{\eta, n}\right| \geq T_{\eta} \\
0, i f\left|d_{\eta, n}\right|<T_{\eta}
\end{array}\right.
$$

Based on the variability of the ionospheric process, $T_{\eta}^{a d}$ adaptive thresholds were introduced, and in Eq. (4) the coefficients $\left\{d_{\eta, n}\right\}_{(\eta, m) \in P_{M}}$ were set to:

$$
d_{\eta, n}=\left\{\begin{array}{l}
d_{\eta, n}^{+}, \text {if }\left(d_{\eta, n}-d_{\eta, n}^{\text {med }}\right) \geq T_{\eta}^{a d} \\
d_{\eta, n}^{-}, \text {if }\left(d_{\eta, n}-d_{\eta, n}^{\text {med }}\right) \leq-T_{\eta}^{a d},
\end{array}\right.
$$

where $T_{\eta}^{a d}=U * S t_{\eta}, S t_{\eta}=\sqrt{\frac{1}{\Phi-1} \sum_{n=1}^{\Phi}\left(d_{\eta, n}-\overline{d_{\eta, n}}\right)^{2}}, \overline{d_{\eta, n}}$ and $d_{\eta, n}^{m e d}$ are the average and the median, respectively, which, in view of the daily course of the ionospheric data, are calculated separately for each time moment $t=n$ in a moving time window of length $\Phi$

Then the $d_{\eta, n}^{+}\left(d_{\eta, n}^{-}\right)$coefficients satisfying the condition (5) determine the time moments $t=n$ in which we observe a local (within the analyzed moving time window) increase (decrease) of the electron concentration for a scale $\eta$ (the deviation from the characteristic level exceeds the $T_{\eta}^{a d}$ threshold).

In the work, the $U$ threshold coefficient (see Eq. (5)) was determined by the criterion of the lowest error rate (a posteriori risk was estimated and minimized [23]). During the estimation of the a posteriori risk in determining the ionospheric conditions, we used $K$ index of geomagnetic activity. The $U$ threshold coefficient was determined separately for the periods of high $\left(f_{10.7}>100\right)$ and low $\left(f_{10.7} \leq 100\right)$ solar activity. For Paratunka station, we estimated the following values of the $U$ coefficient: $2.5 \leq U \leq 3.5$ for the periods of high solar activity, and $1.5 \leq U \leq 2.5$ for low solar activity.

Since the absolute value of the $\left|d_{\eta, n}\right|$ coefficient characterizes the occurred anomalous feature amplitude on a scale $\eta$ (i.e., the value of the function deviation from its characteristic level), it is logical to define it as a measure of ionospheric perturbation on a scale $\eta$ scale. Then the intensity of positive $\left(I^{+}\right)$and negative $\left(I^{-}\right)$ionospheric anomalies at time $t=n$ can be estimated as

$$
I_{n}^{+-}=\sum_{\eta} \frac{\left|d_{\eta, n}^{+-}\right|}{\left\|d_{\eta, n}^{+-}\right\|_{2}}
$$

where $\left\|d_{\eta, n}^{+-}\right\|_{2}=\sqrt{\sum_{n=1}^{N_{\eta}}\left(d_{\eta, n}^{+-}\right)^{2}}, N_{\eta}$ is the series length for scale $\eta$.

Based on the multiscale structure of $\delta_{\text {dist }}^{\eta}(t)$ components, we divide the set of pairs of indices (see Eq. (3)) into two subsets $P_{M_{1}}=\{(\eta, n)\}_{\eta \leq \eta_{1}}$ and $P_{M_{2}}=\{(\eta, n)\}_{\eta>\eta_{1}}, P_{M}=P_{M_{1}} \cup P_{M 2}$. Then the $I(t)$ anomalous component of model (1) will have the following form:

$$
\begin{aligned}
I(t)=\sum_{\eta} \delta_{\text {dist }}^{\eta}(t) & =\sum_{(\eta, n) \in P_{M_{1}}} d_{\eta, n} \Psi_{\eta, n}(t)+\sum_{(\eta, n) \in P_{M_{2}}} d_{\eta, n} \Psi_{\eta, n}(t)+e(t) \\
& =A_{\text {shot }}(t)+A_{\text {long }}(t)+e(t)
\end{aligned}
$$


where $A_{\text {shot }}(t)=\sum_{(\eta, n) \in P_{M_{1}}} d_{\eta, n} \Psi_{\eta, n}(t)$ is a component which describes small-scale anomalous changes occurring for example during solar flares, geomagnetic disturbances, etc., and $\mathrm{A}_{\text {long }}(t)=\sum_{(\eta, n) \in P_{M_{2}}} d_{\eta, n} \Psi_{\eta, n}(t)$ is a component which describes large-scale anomalous changes occurring during long-term ionospheric storms.

Assuming that during long-term anomalous changes $\left(\mathrm{A}_{\text {long }}(t) \neq 0\right)$ the time series structure is changed and, as a sequence, residual errors $a_{j r e g}^{\mu}{ }_{, k}$ of the $A^{R E G}$ regular component of the model (see Eq. (2)) increase. Therefore, identification of the $\mathrm{A}_{\text {long }}(t)$ components can be based on checking the following condition:

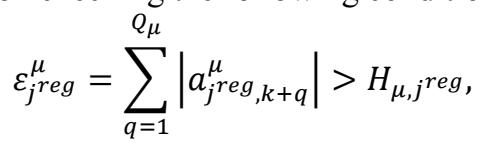

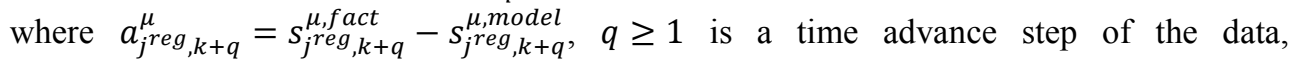

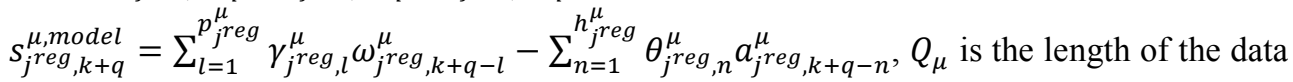
advance time based on the $\mu$-th component model, and $H_{\mu, j}$ reg is the threshold value of the $\mu$-th component defining the presence of anomalous changes in the $\mu$-th component.

Following the paper [16], the $H_{\mu, j}$ reg threshold value in Eq. (7) was determined by:

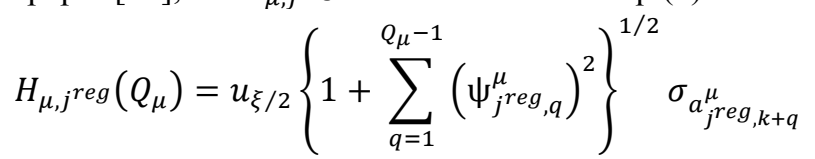

where $u_{\xi / 2}$ is the quantile of the level $(1-\xi / 2)$ of standard normal distribution, $\sigma_{a_{j}^{\mu}{ }^{\mu} g_{, k+q}}$ is variance of residual errors of the $\mu$-th component model, and $\psi_{j}^{\mu}{ }^{r e g}, q$ are the weighting coefficients of the $\mu$-th component model, which can be determined by the relation $[16]$

$$
\begin{aligned}
& \left(1-\varphi_{j}^{\mu}{ }_{r e g, 1} B-\varphi_{j}^{\mu}{ }_{r e g, 2} B^{2}-\cdots-\varphi_{j^{r e g}, p_{j}^{\mu}{ }_{r e g}+v^{\mu}}^{\mu} B^{p_{j^{r e g}}^{\mu}+v^{\mu}}\right)\left(1+\psi_{j}^{\mu} r_{, 1} B+\psi_{j^{r e g}, 2}^{\mu} B^{2}\right.
\end{aligned}
$$

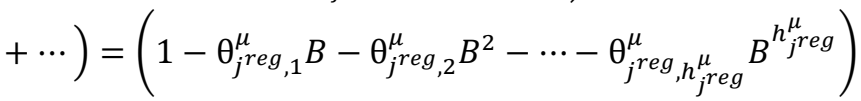

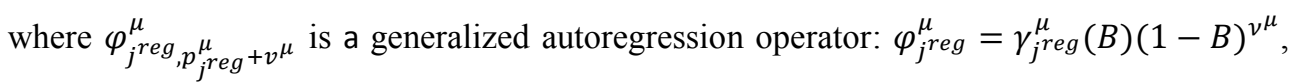

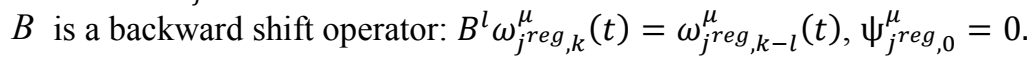

Estimation of the thresholds $H_{\mu, j}$ reg (see Eq. (7)) was carried out using 15-minute data of foF2 on the basis of Eq. (8), (the data of Paratunka station registered during calm conditions were used). We obtained the following results:

1. summer season: $H_{1,-5}=1.9$ is the threshold value for $s_{-5, k}^{1}(t) ; H_{2,-5}=1.3$ is the threshold value for $s_{-5, k}^{2}(t)$

2. winter season: $H_{1,-5}=2.6$ is the threshold value for $s_{-5, k}^{1}(t) ; H_{2,-5}=1.3$ is the threshold value for $s_{-5, k}^{2}(t)$.

\section{Results of experimental studies}

Fig. 1, 2 show the results of the application of the described method (section 2). We used 15-minute (Paratunka, 53.0 N, 158.7 E) and hourly (Wakkanai, 45.16 N, 141.75 E and Moscow, 55.5 N, 37.3 E) data of the ionospheric critical frequency (foF2) for the experiments. 
Figure 1 illustrates the results of the ionospheric data (foF2) processing during the magnetic storm on July 16-20, 2017. According to the cosmic weather review (http://ipg.geospace.ru) on July 12-15, due to the reduction of the influence from the coronal hole, the solar wind speed gradually decreased from 540 to $300 \mathrm{~km} / \mathrm{s}$, the $\mathrm{Bz}$ component of the IMF fluctuated from $\mathrm{Bz}= \pm 2 \mathrm{nT}$ to $\mathrm{Bz}= \pm 4 \mathrm{nT}$. At $05.45 \mathrm{UT}$ on July 16, due to the arrival of an inhomogeneous accelerated flow from the coronal hole and coronal mass ejections, the solar wind speed increased to $660 \mathrm{~km} / \mathrm{s}$ during the day, the fluctuations of the southern component of the IMF increased to $\mathrm{Bz}= \pm 23 \mathrm{nT}$. Then, until the end of the analyzed period, the solar wind speed was within $400-640 \mathrm{~km} / \mathrm{s}$, the fluctuations of the southern component of the IMF varied within $\pm 12 \mathrm{nT}$. The results of the foF 2 data processing show the presence of positive anomalous changes in the ionospheric parameters (an increase in the electron concentration of the ionosphere is shown in Fig. $1 \mathrm{~b}, \mathrm{e}, \mathrm{h}$ in red, Eq. (5)) occurring on the eve of the magnetic storm in the analyzed areas (the pre-increase effect in the ionosphere $[3,7,8,9])$. At Paratunka and Moscow stations, positive anomaly occurred about 35-37 hours before the onset of the magnetic storm, at Wakkanai station about 15 hours. The analysis of GMCM residual errors (Fig. 1 d, j, Eq. (7)) also indicates the presence of anomalous changes in the ionospheric data time course on the eve of the event (the exceedance of the confidence intervals (STD) (Eq. (8)): the exceedance of 2.2 STD for Paratunka station and the exceedance of 1.6 STD for Wakkanai station). In contrast with GMCM, the application of the median method (Fig. 1a) did not make it possible to detect the anomalous change in the ionospheric parameters. During the initial phase of the magnetic storm, the electron concentration in the ionosphere was also anomalously increased (positive anomaly is shown in Fig. $1 \mathrm{~b}$, e, h; significant deviations of the current data values from the median values (Fig. 1a)), which was probably due to the effect of rapid penetration of the electric field into the middle and low latitudes (PPEF effect [24]). At the recovery phase, the decrease in the electron concentration (negative anomaly is shown in Fig. $1 \mathrm{~b}$, e, h in blue) began at Paratunka and Moscow stations with a difference of several hours and lasted for about 26 hours (there were gaps in the data at Paratunka station from 10:15 UT to $23: 45$ UT on July 17, 2017). The negative anomaly is probably due to the heating and elevation of the thermosphere, which leads to an increase of the recombination rate and, consequently, to depletion of ionization [2]. The duration of the negative anomaly was about 9 hours at Wakkanai station (Fig. $1 \mathrm{~h}$ ). The modeling results (Fig. $1 \mathrm{~d}, \mathrm{~g}, \mathrm{j}$ ) indicate significant changes in the foF2 time course during negative phase of ionospheric storm (3.95 STD at Paratunka station, 2.7 STD at Wakkanai station and 3.3 STD at Moscow station) and its prolonged restoration (about 44 hours at Paratunka station and about 38 hours at Wakkanai and Moscow stations). Analogous dynamics of the ionospheric parameters during magnetic storms was repeatedly noted earlier in the papers $[4,25]$. 

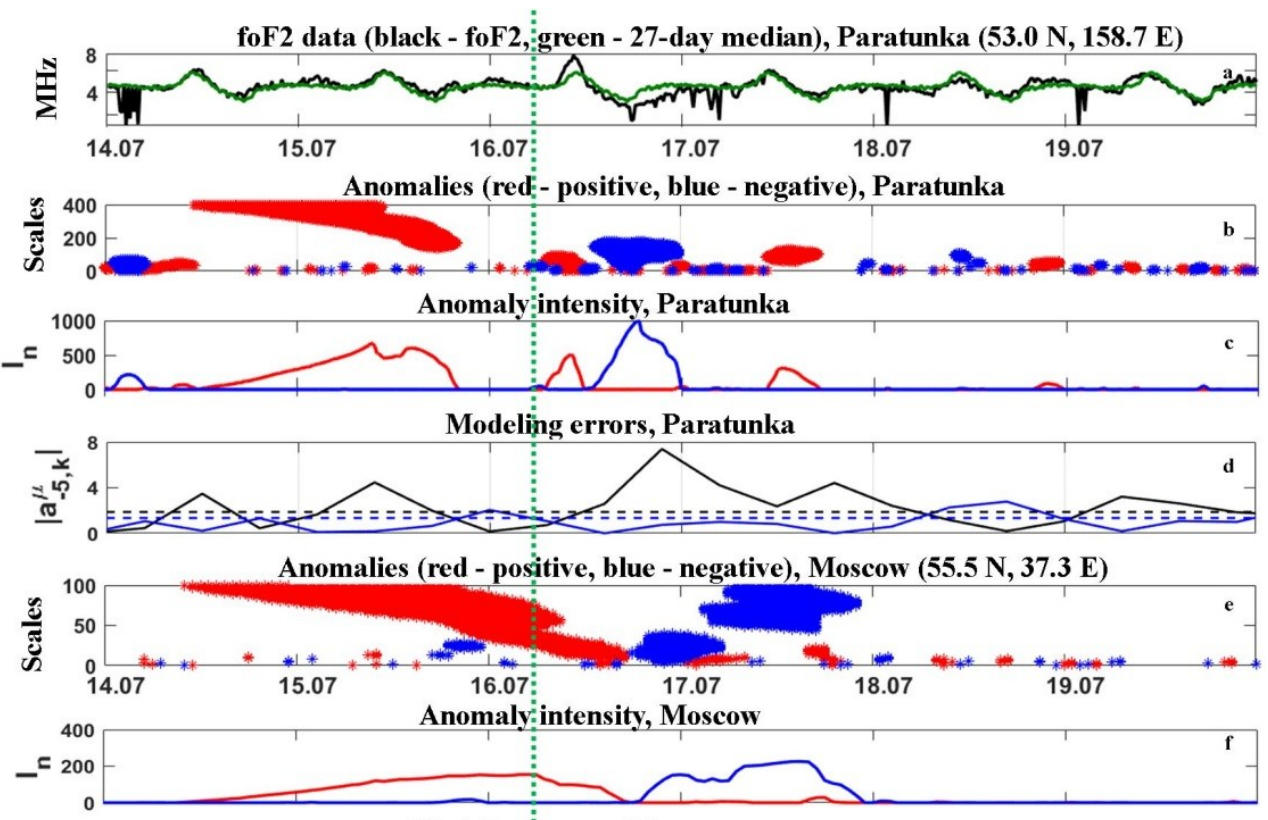

Modeling errors, Moscow
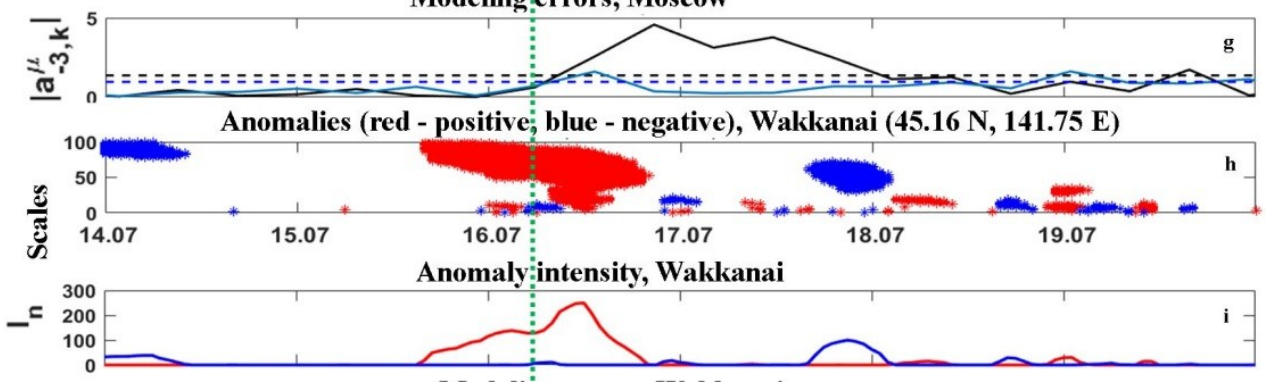

Modeling errors, Wakkanai
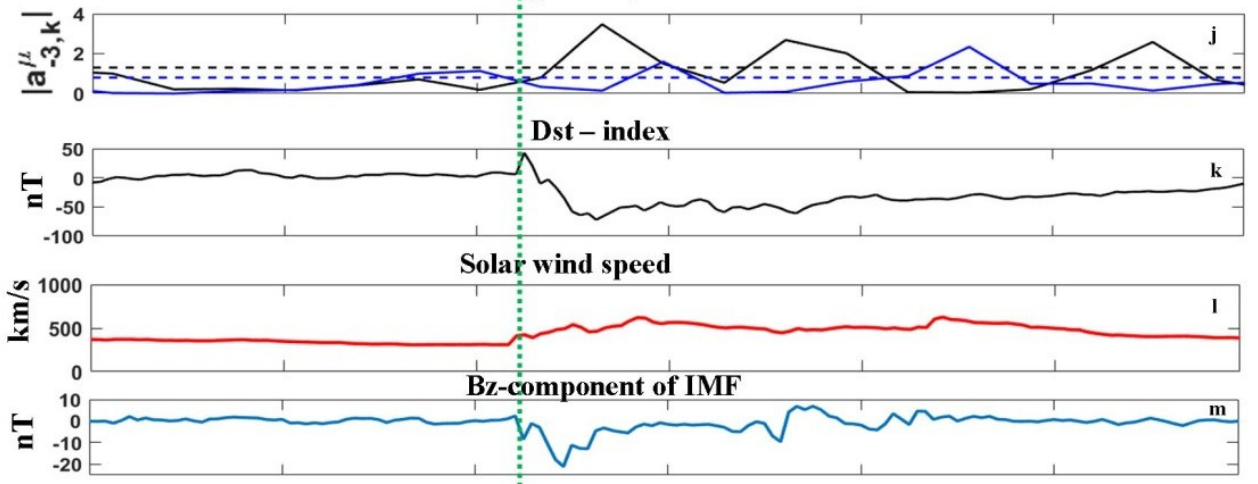

Fig. 1. Results of the ionospheric critical frequency data (foF2) processing for July $14-19$, 2017 (time UT): d), g), j) - the confidence intervals of GMCM are shown by dashed lines (confidence probability is $70 \%$ ). Green dashed line is the beginning of the storm.

Fig. 2, 3 show the results of the ionospheric data (foF2) processing during the magnetic storm on April 20, 2018. According to the cosmic weather review (http://ipg.geospace.ru) during the day on April 18-19, the solar wind speed was within $280-313 \mathrm{~km} / \mathrm{s}$, the IMF $\mathrm{Bz}$ component fluctuated within $\pm 5 \mathrm{nT}$. At the end of the day of April 19, an 
inhomogeneous accelerated flow arrived from a coronal hole (CIR). During the day on April 20, the solar wind speed increased to $650 \mathrm{~km} / \mathrm{s}$ and remained within $520-650 \mathrm{~km} / \mathrm{s}$ until the end of the day on April 21, fluctuations of the IMF southern component increased to $\mathrm{Bz}= \pm 19 \mathrm{nT}$ at $08.12 \mathrm{UT}$ on April 20. Due to the reduction of the influence from the coronal hole, the solar wind speed gradually decreased to $350 \mathrm{~km} / \mathrm{s}$, the fluctuations of the IMF southern component decreased to $\mathrm{Bz}= \pm 5 \mathrm{nT}$. The ionosphere behavior during the magnetic storm demonstrates a similar character with the previous event. Fig. 2 shows the results of data processing using the threshold coefficient $U=2.5$ (the exceedance of 2.5 STD in the analyzed time window, see Eq. (5), Fig. 2 b, c, e, f, h, i). Analysis shows that on the eve of the magnetic storm (from 20:00 UT on April 18 to 05:00 UT on April 19), the level of electron concentration decreased at Wakkanai station (Fig. 2e), and increased at Moscow station (Fig. 2h). At Paratunka and Wakkanai stations, an increase in the electron concentration is observed about 2 hours before the beginning of the magnetic storm (Fig. $2 \mathrm{~b}, \mathrm{e}$, it is shown in red), which reached its maximum intensity during the main phase of the magnetic storm (Fig. 2c, f). At Moscow station during the initial and main phases of the magnetic storm, the ionospheric concentration was also increased (see Fig. 2 h, i). Then, the electron concentration significantly decreased (Fig. 2b, e). The negative anomaly that occurred during the recovery phase reached the maximum values at the analyzed stations during the period from 14:00 to 18:00 UT on April 21, 2018 (Fig. 2c, f). The modeling results (Fig. 2d, g, j) show that a decrease in the electron concentration led to a significant change in the time course of the foF 2 data, as evidenced by an increase of GMCM errors (the exceedance of 4.8 STD at Paratunka station, 3.6 STD at Wakkanai station and 2.3 STD at Moscow station) and significant deviations of the data from the median (Fig. 2a). Fig. 3 shows the results of ionospheric data processing using the threshold coefficient $U=2$ (the exceedance of 2 STD in the analyzed time window). Analysis of the results confirms the dynamics of the ionospheric process at the analyzed stations described above and shows the efficiency of the proposed method. The possibility of adaptive tuning of the algorithms makes it possible to study the dynamics of the ionospheric parameters in detail and to estimate the moments of the onset of ionospheric disturbances with high accuracy. 


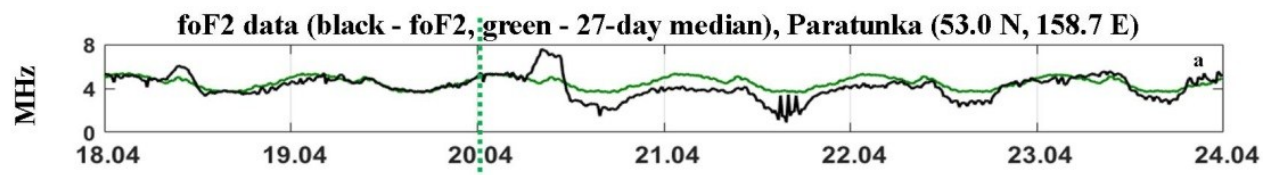

Anomalies (rểd - positive, blue - negative), Paratunka
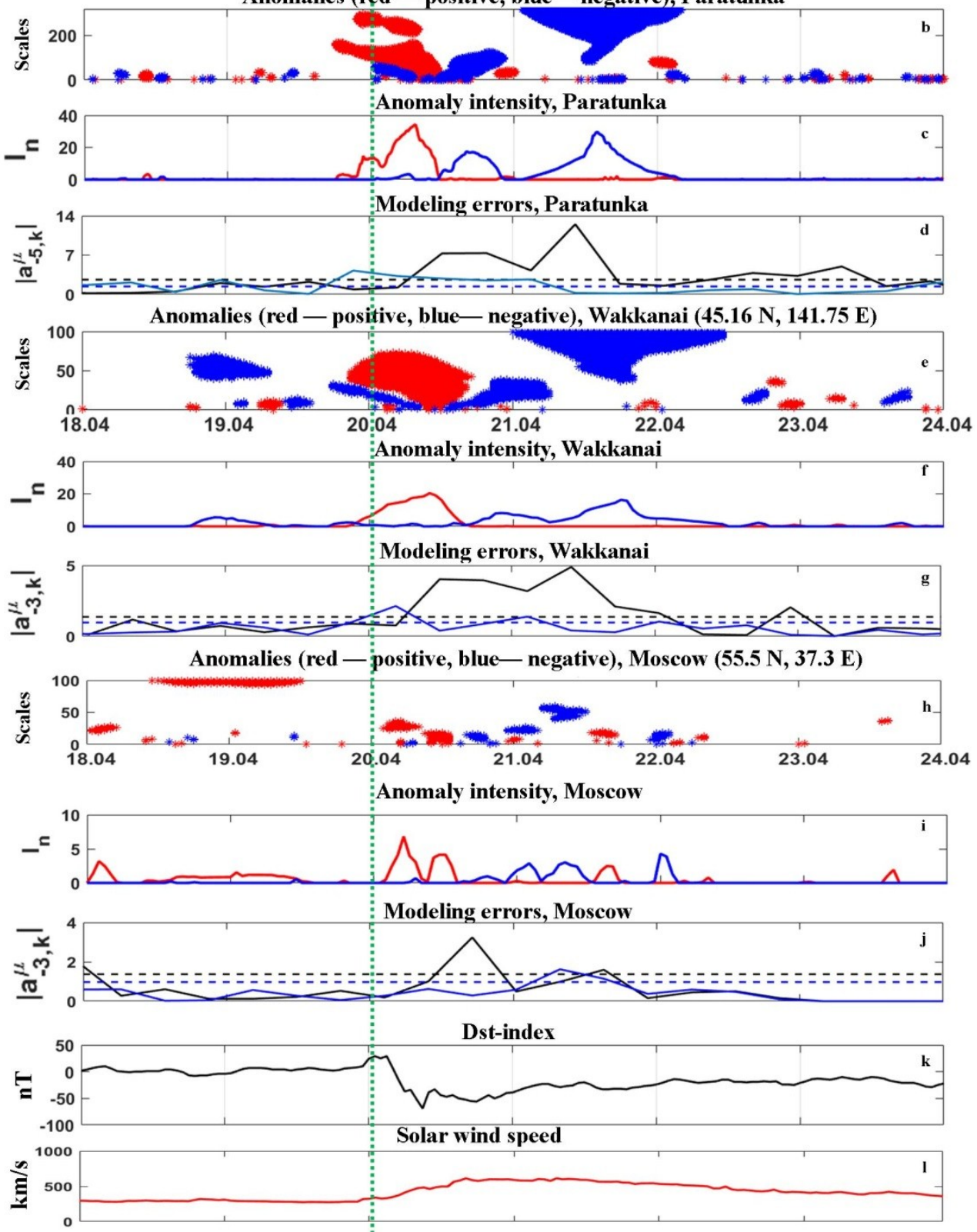

Fig. 2. Results of the ionospheric critical frequency data (foF2) processing for April 18 23, 2018 (time UT): d), g), j) - the confidence intervals of GMCM are shown by dashed lines (confidence probability is $70 \%$ ). Green dashed line is the beginning of the storm. 


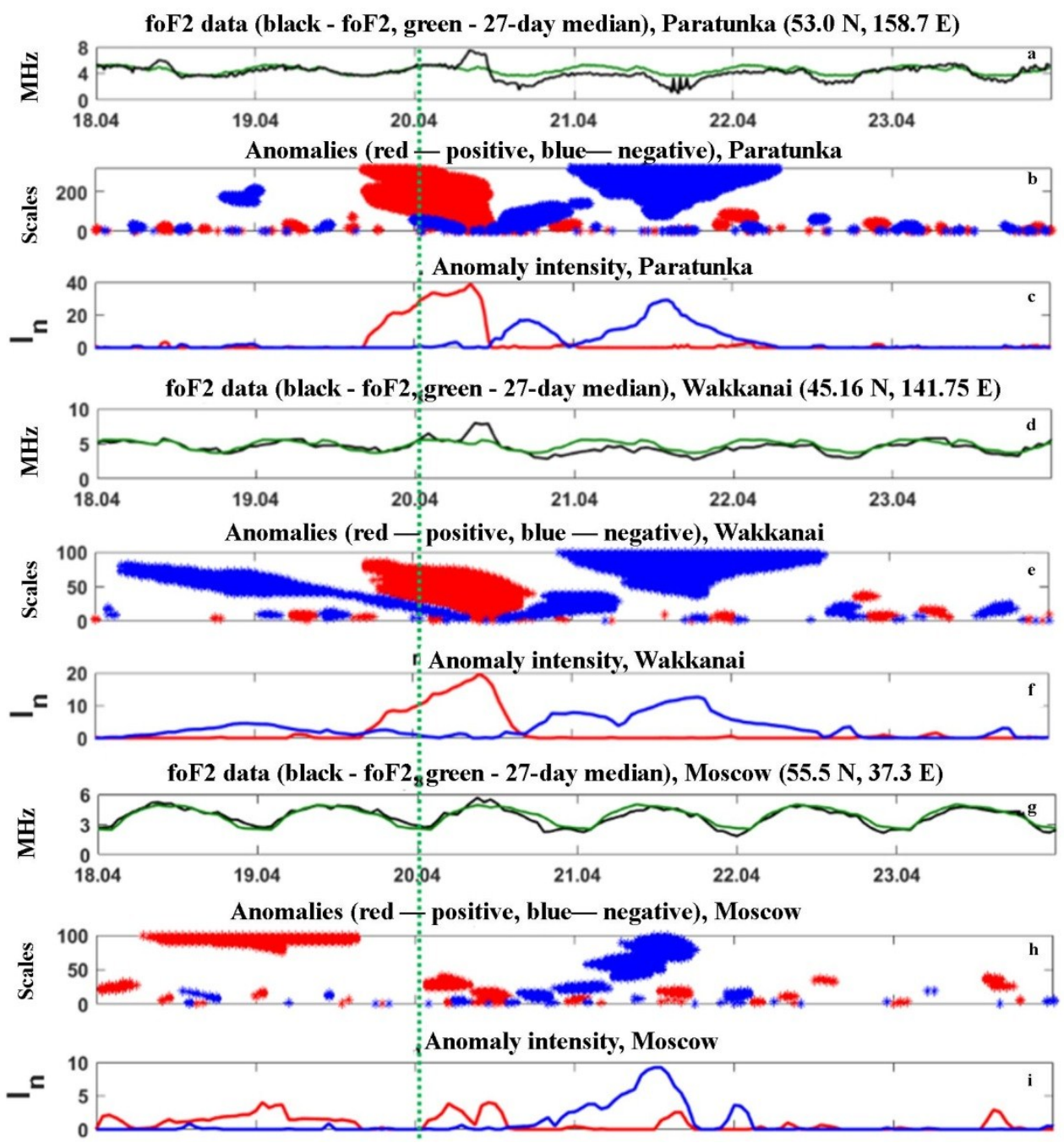

Fig. 3. Results of the ionospheric critical frequency data (foF2) processing for April 18 23, 2018 (time UT, the threshold coefficient $U=2$ ). Green dashed line is the beginning of the storm.

\section{Conclusions}

The application of GMCM allowed us to study the dynamics of the ionospheric parameters during the disturbed periods in detail. A similar behavior of the ionosphere was observed in the analyzed regions, an anomalous increase in the electron concentration of the ionosphere several hours before the beginning of the magnetic storms (the pre-increase effect in the ionosphere [3, 7]). During the initial phase, the electron concentration also remained anomalously increased, and during the recovery phase, there was a strong decrease in the electron concentration, and negative ionospheric storms, leading to a significant and prolonged change in the time course of the ionospheric process, occurred. According to the results of the papers [27, 28], a similar behavior of the ionosphere is typical for the periods of increased solar activity and can be observed in regions from high 
to low latitudes. The long-term negative ionospheric storms occurring during recovery phase are probably due to the heating and elevation of the thermosphere, which lead to an increase of the recombination rate and, consequently, to depletion of ionization [29]. Anomalous increases detected in the ionosphere on the eve of the magnetic storms have been repeatedly observed in the works $[3,4,7]$ and have been analyzed using GMCM in the papers $[8,19,28]$ in more detail. The observed frequency of the pre-increase effect at different stations and the comparison with the parameters of the interplanetary medium allow us to make an assumption about the validity of the hypothesis presented in the paper [3] and to suppose that such ionospheric anomalies are associated with a certain channel of energy penetration from interplanetary space and the magnetosphere. In this case, such effects are of interest in space weather problems and can serve as a indicator for an approaching magnetic storm. According to the results of this study, the detection of these effects can be performed on the basis of GMCM, and the efficiency of this approach can be improved if the number of analyzed recording data stationsis extended.

In the future on the basis of the developed approach, the authors plan to continue studying the ionospheric parameter dynamics applying a wider range of data recording stations and increasing the statistics.

The research is supported by a grant from the Russian Science Foundation (project No. 14-11-00194). The authors are grateful to the organizations recording the data which were applied in the paper. The work was carried out by the means of the Common Use Center "North-Eastern Heliogeophysical Center" CKP_558279.

\section{References}

1. E.L. Afraimovich, N.P. Perevalova, GPS-monitoring verhnejatmosferyZemli [GPSmonitoring of the Earth's upper atmosphere] (GU NU RVH VSNC SO RAMN, Irkutsk, 2006)

2. M. Nakamura, T. Maruyama, Y. Shidama, Journal of the National Institute of Information and Communications Technology, 56, 391-406 (2009)

3. A.D. Danilov, Advances in Space Research, 52, 343-366 (2013)

4. O.V. Mandrikova, N.V. Fetisova, Y.A. Polozov, I.S. Solovev, M.S. Kupriyanov, Earth, Planets and Space, 67, (2015)

5. M. I. Panasyuk et al., Cosmic Research, 42, 489-535 (2003)

6. D.G. Baishev, A.V. Moiseyev, R.N. Boroyev, S.E. Kobyakova, A.E. Stepanov, O.V. Mandrikova, I.S. Solovev, S.Yu. Khomutov, Yu.A. Polozov, A. Yoshikawa., K. Yumoto, Sun and Geosphere, 10, 133-140 (2015)

7. D.V. Blagoveshchensky, A. S. Kalishin, Geomagnetism and Aeronomy, 11, 200-209 (2009)

8. O.V. Mandrikova, N.V. Fetisova, Yu.A. PolozovProceedings of the 11th Intl School and Conference "Problems of Geocosmos", 262-269 (2017)

9. O.V. Mandrikova, Yu.A. Polozov, N.V. Fetisova, T.L. Zalyaev Journal of Atmospheric and Solar-Terrestrial Physics (to be published)

10. N. I. Budko, A.N. Zaitsev, A.T. Karpachev, A.N. Kozlov, B.P. Filippov, Kosmicheskayasredavokrugnas. [The space environment around us] (TROVANT, Troitsk, 2005)

11. Y. Kakinami, J. Liu, L. Tsai, K. Oyama International Journal of Remote Sensing, 31, 3571-3578 (2010)

12. D. V. Solomentsev, B. V. Khattatov, A.A. Titov, Geomagn. Aeron., 53, 73-84 (2013)

13. D. Bilitza, B.W. Reinisc, Advances in space research, 42, 599-609 (2007)

14. V.N. Shubin, A.T. Karpachev, V.A. Telegin, K.G. Tsybulya, Geomagnetism and Aeronomy, 55, 609-622 (2015) 
15. X. Zhao, B. Ning, L. Liu, G. Song, Advances in Space Research, 53, 387-394 (2014)

16. G. Box, G. Jenkins, Time series analysis: Forecasting and control (Holden-Day, San Francisco, 1970)

17. G.P. Anshakov, A.V. Raschupkin, Y.N. Zhuravel, Computer Optics, 39, 77-82 (2015)

18. A.Y. Denisova, Y.N. Juravel, V.V. Myasnikov, Computer Optics, 40, 380-387 (2016)

19. O. Mandrikova, N. Fetisova, Yu. Polozov, Journal of Physics: Conference Series by IOP Publishing (to be published)

20. S. Mallat, A wavelet tour of signal processing (Academic Press, London, 1999)

21. Mandrikova O.V., Glushkova N.V., Zhivetev I.V. Geomagnetism and Aeronomy, 54, № 5, 593-600 (2014)

22. O.V. Mandrikova, Yu.A. Polozov, V.V. Bogdanov, V.V. Geppener, Analiz i interpretaciyageofizicheskihparametrovnaosnovemnogokomponentnyhmodelej.

[Analysis and interpretation of geophysical parameters on the basis of multicomponent models] (Dal'nauka, Vladivostok, 2013)

23. B.R. Levin. Theoretical Foundations of Statistical Radio Engineering. [Teoreticheskieosnovystatisticheskoyradiotekhniki] (Sov.radio, Moscow, 1975)

24. M. A. Abdu, J. Atmos. Sol. Terr. Phys., 59, 1505-1519 (1997)

25. O.V. Mandrikova, I.S. Solovjev, S.Yu. Khomutov, D.G. Baishev, V.V. Geppener, D.M. Klionskiy, Pattern recognition and image analysis, 26, (2016)

26. O. Mandrikova, Yu. Polozov, V. Geppener, Procedia Engineering, 201, 756-766 (2017)

27. O.V. Mandrikova, Y.A. Polozov, T.L. Zalyaev, D.G. Baishev, B.M. Shevtsov, AOGS 14th Annual Meeting (2017)

28. O. V. Mandrikova, N. V. Fetisova, Yu. A. Polozov, Japan Geoscience Union Meeting (2018)

29. M. Nakamura, T. Maruyama, Y. Shidama, Earth, Planets Sp., 592008 